Reprod. Nutr. Dévelop. 1980, 20 (4 A), 923-928.

\title{
Note sur les relations entre le rythme cardiaque ef le type de croissance chez la poule domestique
}

par J. M. FAURE, F. H. RICARD

avec la collaboration technique de S. CORNU, et G. MARCHE

Station Expérimentale d'Aviculture du Magneraud, I.N.R.A., B. P. $n^{0} 52,17700$ Surgères, France.

Summary. A note on the relationship between heart rate and growth pattern in the domestic fowl.

The heart rate at 6 and 24 weeks of age and the relative weight of the heart at 8 and 24 weeks were studied in four strains of domestic fowl, differing in growth and, in this study, in body weight.

Heart rate at 6 weeks of age was not related to sex, body weight, absolute or relative heart weight. Heart rate at 24 weeks of age was related to relative heart weight at 24 weeks, to absolute heart weight at 6 and 24 weeks, and to body weight at 6 weeks. There was a marked sex effect at 24 weeks.

No within-species relationship between heart rate and body weight was found, but a negative correlation existed between heart rate and relative heart weight.

Les oiseaux ou mammifères de petite taille ont généralement un rythme cardiaque plus élevé que celui des animaux de grande taille, bien que cette règle présente des exceptions (cf. Sturkie, 1965, tabl. p. 124). On observe également un dimorphisme sexuel qui a parfois été attribué à une différence de taille (Cadieu, 1974, 1976). Cette relation entre poids corporel et rythme cardiaque a cependant surtout été étudiée au niveau interspécifique et peu de choses sont connues au niveau intraspécifique même dans une espèce aussi polymorphe que la poule. Dans la présente étude, nous avons utilisé des souches de poules sélectionnées sur la forme de leur courbe de croissance (Ricard, 1975) pour voir quelle pouvait être l'influence du poids juvénile et du poids adulte sur le rythme cardiaque d'animaux jeunes et pré-adultes, et si les relations poids-fréquence cardiaque observées au nivequ interspécifique se retrouvent entre des souches d'origine commune d'une même espèce polymorphe. 


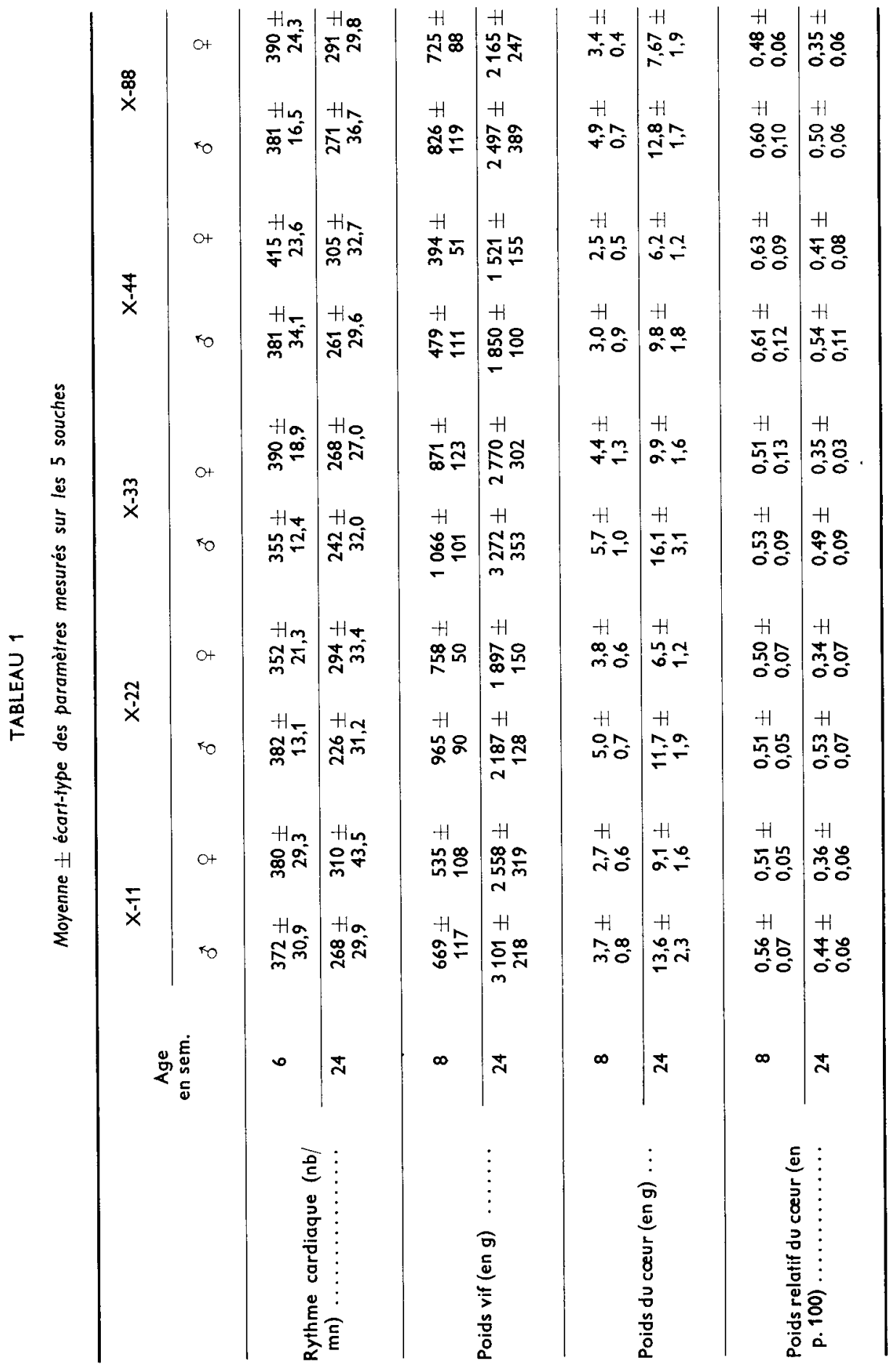




\section{Matériel et méthodes.}

A partir d'une souche de type Bresse-pile maintenue sans sélection comme témoin, quatre souches ont été sélectionnées sur la forme de leur courbe de croissance (Ricard, 1975) pour obtenir les combinaisons suivantes :

Souche $X-11$ : poids à 8 semaines faible et poids adulte fort.

Souche X-22: $\quad$ - fort faible.

Souche X-33 : - fort - $\quad$ fort.

Souche X-44: $\quad-$ faible $\quad-$ faible.

Souche $X-88$ : témoin non sélectionné.

Le rythme cardiaque a été déterminé à deux âges différents ( 6 et 24 semaines) sur 6 animaux par souche et par sexe. Ces animaux ont été choisis aux alentours du poids vif moyen de leur groupe. La mesure a été faite sur l'animal vivant en contention manuelle, ce qui peut se justifier par le fait qu'il y a peu de différence entre la mesure sur l'animal libre au repos et celle effectuée sur l'animal en contention (Sturkie, 1963). L'enregistrement électrocardiographique a été effectué sur table déroulante SEFRAM à la vitesse de 2,5 mm par seconde. Les électrodes utilisées sont des aiguilles à injection dont l'une est plantée sous la peau du dos au niveau de la ceinture pelvienne et l'autre sous l'aile droite au niveau de l'humérus. Ce type d'implantation a été choisi car une expérience préliminaire avait montré qu'il permettaif de recueillir un tracé de bonne qualité et qu'il ne semblait pas perturber l'animal libre. Puis, pour chaque animal, le nombre de battements cardiaques a été compté sur une période d'une minute.

A l'occasion d'une autre expérience portant sur les mêmes souches, le poids vif et le poids du cœur avaient été mesurés aux âges de 8 et 24 semaines, sur 10 animaux par souche et par sexe. Ces données ont été reprises en calculant le poids du cœur relativement au poids vif.

Les résultats des groupes souche-sexe ont été comparés globalement par la méthode de l'analyse de variance. Les facteurs étudiés étaient : le poids juvénile (fort ou faible), le poids adulte (fort ou faible) et le sexe. Dans ces analyses, la souche témoin X-88 n'était pas incluse. La relation entre rythme cardiaque et poids relatif du coeur a été précisée en calculant une corrélation sur les moyennes de souche, une souche étant donc considérée ici comme un individu.

\section{Résultats ef discussion.}

Les valeurs moyenne et les écarts-types des caractères mesurés sont indiqués dans le tableau 1. Le poids vif des animaux abattus donne une bonne indication du type de croissance des souches utilisées. Le rythme cardiaque observé à 6 semaines est plus faible que celui cité par Sturkie (1965) pour des poulets de race Leghorn. II est vrai que nos animaux sont, en moyenne, plus lourds. A 24 semaines, le rythme observé dans nos souches est comparable à celui des animaux Rhode-Island ef inférieur à celui des Leghorn, en se fiant également aux chiffres cités par Sturkie.

Une remarque intéressante concerne la variabilité du rythme cardiaque en fonction de l'âge : les coefficients de variation intra-groupes sont toujours plus faibles chez les jeunes $(<10$ p. 100) que chez les adultes ( $>10$ p. 100).

Les analyses de variance sont résumées dans le tableau 2 . Les corrélations entre 


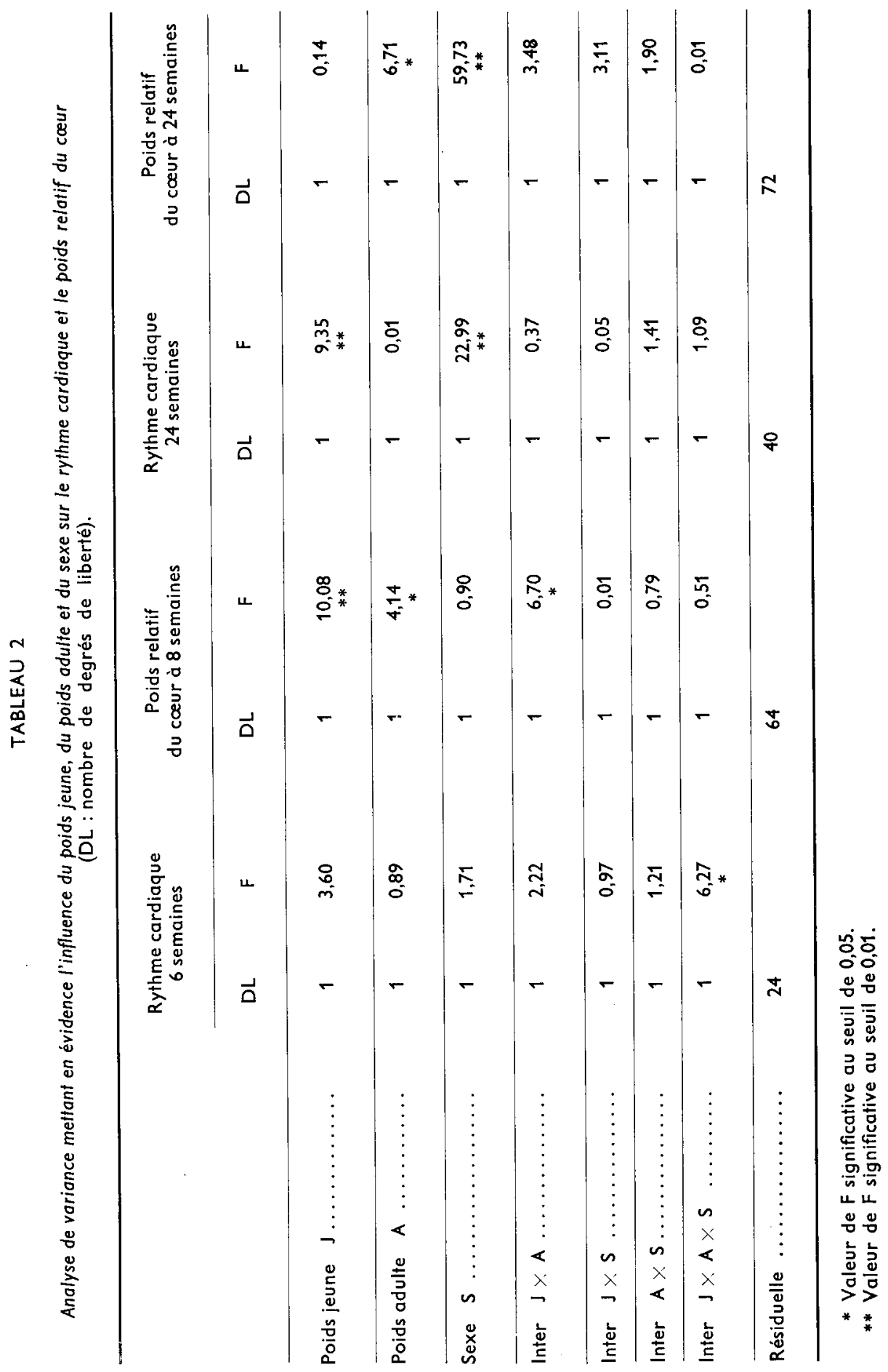


moyennes de souches pour le rythme cardiaque et le poids relatif du cœur (par rapport au poids vif) sont indiquées dans le tableau 3.

\section{TABLEAU 3}

Corrélation entre le rythme cardiaque à 6 ef 24 semaines d'âge et les poids relatifs du cœur (Poids rel. cœur), les poids du cœur (Poids cœur) ef les poids du corps (Poids).

Les calculs ont été effectués sur les moyennes de souche.

\begin{tabular}{lcccccc}
\hline & $\begin{array}{c}\text { Poids rel. } \\
\text { cœur } \\
8 \mathrm{sem} .\end{array}$ & $\begin{array}{c}\text { Poids rel. } \\
\text { cour } \\
24 \mathrm{sem} .\end{array}$ & $\begin{array}{c}\text { Poids } \\
\text { cour } \\
8 \mathrm{sem} .\end{array}$ & $\begin{array}{c}\text { Poids } \\
\text { cœur } \\
24 \text { sem. }\end{array}$ & $\begin{array}{c}\text { Poids } \\
8 \mathrm{sem} .\end{array}$ & $\begin{array}{c}\text { Poids } \\
24 \text { sem. }\end{array}$ \\
\hline Rythme 6 semaines $\ldots .$. & 0,45 & $-0,07$ & $-0,50$ & $-0,45$ & $-0,54$ & $-0,48$ \\
\hline Rythme 24 semaines ... & 0,03 & $-0,74$ & $-0,77$ & $-0,72$ & $-0,70$ & $-0,39$ \\
\hline
\end{tabular}

L'analyse de variance du rythme cardiaque à 6 semaines montre qu'aucun des facteurs testés (sexe, poids juvénile, poids adulte) n'a un effet significatif au seuil 5 p. 100. Toutefois, le poids juvénile a un effet significatif au seuil 10 p. 100, les animaux les plus lourds ayant le trythme le plus lent. L'intéraction triple est significative, ce qui pourrait s'expliquer par le fait que dans la souche $X-22$ les femelles ont un rythme significativement plus lent que celui des mâles $(P<0,05)$ alors que c'est l'inverse dans toutes les autres souches (significatif seulement pour la souche $X-33, P<0,01$ ). Les corrélations sur les moyennes de souches faisant intervenir le rythme 6 semaines ne sont pas significatives, mais on observe la relation rythme lent associé à un poids juvénile élevé et à poids du cœur élevé.

A 24 semaines d'âge, l'analyse de variance montre que le poids jeune et le sexe influencent de manière significative le rythme cardiaque. Les animaux ayant un poids juvénile faible ont un rythme adulte plus élevé et, comme le constate Sturkie, les poules ont une fréquence cardiaque plus élevée que celle des coqs. II n'y a par contre pas d'effet du poids du corps à l'âge adulte.

Les corrélations entre le rythme cardiaque adulte moyen et les moyennes du poids du cœur à 6 et 24 semaines, le poids relatif du cœur à 24 semaines ef le poids vif à 8 semaines sont toutes significatives. Les corrélations avec le poids relatif du cœur à 8 semaines ef le poids vif à 24 semaines sont par contre non significatives.

D'après ces données, on peut donc dire que le rythme cardiaque du jeune poulet ne dépend ni du sexe, ni du type de croissance. Par contre, à 24 semaines d'âge, c'est-à-dire au début de la ponte pour les femelles, l'effet dô au sexe est très net. Le rythme dépend aussi du poids juvénile, comme si l'activité physiologique du cœur était programmée à partir du développement corporel avant la puberté.

Parmi les oiseaux ou les mammifères, plusieurs auteurs ont trouvé une corrélation négative entre le rythme cardiaque et le poids du corps (Sturkie, 1965), et une corrélation positive entre le poids relatif du cœur et le rythme cardiaque (Portmann, 1950). Il n'en est pas de même à l'intérieur d'une espèce puisque dans les souches de poules sélectionnées pour la forme de leur courbe de croissance, nous trouvons qu'il 
n'y a pas de relation entre le poids du corps ef le rythme cardiaque, mais qu'il y a par confre une corrélation négative entre le poids relatif du cœur et le rythme cardiaque. La relation fréquemment observée au niveau interspécifique ne se retrouve donc pas au niveau intraspécifique si l'on étudie un ensemble de souches d'origine commune mais bien différenciées au niveau du poids.

Reçu en juillet 1979.

Accepté en novembre 1979.

\section{Références}

CADIEU N., 1974. Contribution ò l'éfude des influences délètères de la consanguinité sur le comportement de la caille japonaise (Coturnix coturnix japonica). Th. $3^{\text {e }}$ cycle, Fac. Sci., Toulouse.

CADIEU N., 1976. La réactivité cardiaque au bruit et son atténuation par l'apprivoisement chez la caille japonaise : effets dépressifs de la consanguinité. Biol. Behav., 1, 232-249.

PORTMANN A., 1950. Les organes de la circulation sanguine. In P. P. GRASSÉ Traité de Zoologie, Vol XV. Oiseaux. Masson. Paris.

RICARD F. H., 1975. Essai de sélection sur la forme de la courbe de croissance chez le poulet. Ann. Génét. Sél. anim., 7, 427-443.

STURKIE P. D., 1963. Heart rate of chickens defermined by radiotelemetry during light and dark periods. Poultry Sci., 42, 797-798.

STURKIE P. D., 1965. Avian physiology. Cornell Univ. Press, New-York. 